Address correspondence to: Joseph L. Witztum, Department of Medicine, University of California San Diego, 9500 Gilman Drive, La Jolla, California 92093, USA. Phone: (858) 534-4347; Fax: (858) 534-2005; E-mail: jwitztum@ucsd.edu.

1. Moore, K.J., et al. 2005. Loss of receptor-medi ated lipid uptake via scavenger receptor A or CD36 pathways does not ameliorate atherosclerosis in hyperlipidemic mice. J. Clin. Invest. 115:2192-2201. doi:10.1172/JCI24061.

2. Suzuki, H., et al. 1997. A role for macrophage scavenger receptors in atherosclerosis and susceptibility to infection. Nature. 386:292-296.

3. Babaev, V.R., et al. 2000. Reduced atherosclerotic lesions in mice deficient for total or macrophagespecific expression of scavenger receptor-A. Arterioscler. Thromb. Vasc. Biol. 20:2593-2599.

4. Febbraio, M., et al. 2000. Targeted disruption of the class B scavenger receptor CD36 protects against atherosclerotic lesion development in mice. J. Clin. Invest. 105:1049-1056.

5. Febbraio, M., Guy, E., and Silverstein, R.L. 2004 Stem cell transplantation reveals that absence of macrophage CD36 is protective against atherosclerosis. Arterioscler. Thromb. Vasc. Biol. 24:2333-2338.

6. Freigang, S., Hörkkö, S., Miller, E., Witztum, J.L., and Palinski, W. 1998. Immunization of LDL receptor-deficient mice with homologous malondialdehyde-modified and native LDL reduces progression of atherosclerosis by mechanisms other than induction of high titers of antibodies to oxidative neoepitopes. Arterioscler. Thromb. Vasc. Biol. 18:1972-1982.

7. Skalen, K., et al. 2002. Subendothelial retention of atherogenic lipoproteins in early atherosclerosis.
Nature. 417:750-754.

8. VanderLaan, P.A., Reardon, C.A., and Getz, G.S 2004. Site specificity of atherosclerosis: site-selective responses to atherosclerotic modulators [review]. Arterioscler. Thromb. Vasc. Biol. 24:12-22.

9. Van Eck, M., Bos, I.S., Hildebrand, R.B., Van Rij, B.T., and van Berkel, T.J. 2004. Dual role for scavenger receptor class B, type I on bone marrow-derived cells in atherosclerotic lesion development. Am. J. Pathol. 165:785-794.

10. Herz, J., and Hui, D.Y. 2004. Lipoprotein receptors in the vascular wall. Curr. Opin. Lipidol. 15:175-181

11. Greaves, D.R., and Gordon, S. 2005. Thematic review series: the immune system and atherogenesis. Recent insights into the biology of macrophage scavenger receptors. J. Lipid Res. 46:11-20.

12. Yoshida, H., Quehenberger, O., Kondratenko, N., Green, S., and Steinberg, D. 1998. Minimally oxidized low-density lipoprotein increases expression of scavenger receptor A, CD36, and macrosialin in resident mouse peritoneal macrophages. Arterioscler. Thromb. Vasc. Biol. 18:794-802.

13. Miller, Y.I., et al. 2003. Minimally modified LDL binds to CD14, induces macrophage spreading via TLR4/MD-2, and inhibits phagocytosis of apoptotic cells. J. Biol. Chem. 278:1561-1568.

14. Liang, C.P., et al. 2004. Increased CD36 protein as a response to defective insulin signaling in macrophages. J. Clin. Invest. 113:764-773. doi:10.1172/ JCI200419528.

15. Li, A.C., and Glass, C.K. 2002. The macrophage foam cell as a target for therapeutic intervention. Nat. Med. 8:1235-1242.

16. Kunjathoor, V.V., et al. 2002. Scavenger receptors class A-I/II and CD36 are the principal receptors responsible for the uptake of modified low density lipoprotein leading to lipid loading in macrophages. J. Biol. Chem. 277:49982-49988.
17. Khoo, J.C., Miller, E., Pio, F., Steinberg, D., and Witztum, J.L. 1992. Monoclonal antibodies against LDL further enhance macrophage uptake of LDL aggregates. Arterioscler. Thromb. Vasc. Biol. 12:1258-1266

18. Schissel, S.L., et al. 1998. Secretory sphingomyelinase, a product of the acid sphingomyelinase gene, can hydrolyze atherogenic lympoproteins at neutral $\mathrm{pH}$. Implications for atherosclerotic lesion development. J. Biol. Chem. 273:2738-2746.

19. Griffith, R.L., Virella, G.T., Stevenson, H.C., and Lopes-Virella, M.F. 1988. Low density lipoprotein metabolism by human macrophages activated with low density lipoprotein immune complexes. A possible mechanism of foam cell formation. J. Exp. Med. 168:1041-1059.

20. Yeh, M., et al. 2004. Role for sterol regulatory element-binding protein in activation of endothelial cells by phospholipid oxidation products. Circ. Res. 95:780-788.

21. Kruth, H.S., et al. 2005. Macropinocytosis is the endocytic pathway that mediates macrophage foam cell formation with native low density lipoprotein. J. Biol. Chem. 280:2352-2360.

22. Lindqvist, P., Ostlund-Lindqvist, A.M., Witztum, J.L., Steinberg, D., and Little, J.A. 1983. The role of lipoprotein lipase in the metabolism of triglyceride-rich lipoproteins by macrophages. J. Biol. Chem. 258:9086-9092.

23. Tall, A.R. 2003. Role of ABCA1 in cellular cholesterol efflux and reverse cholesterol transport. Arterioscler. Thromb. Vasc. Biol. 23:710-711.

24. Hoebe, K., et al. 2005. CD36 is a sensor of diacylglycerides. Nature. 433:523-527.

25. Kunjathoor, V.V., Tseng, A.A., Medeiros, L.A., Khan, T., and Moore, K.J. 2004. beta-Amyloid promotes accumulation of lipid peroxides by inhibiting CD36mediated clearance of oxidized lipoproteins. J. Neuroinflammation. 1:23. doi:10.1186/1742-2094-1-23.

\title{
Weaving $\beta$ Klotho into bile acid metabolism
}

\author{
Antonio Moschetta ${ }^{1}$ and Steven A. Kliewer,1,2
}

1Department of Pharmacology and 2Department of Molecular Biology, University of Texas Southwestern Medical Center, Dallas, Texas, USA.

Bile acids are natural detergents that assist in the absorption and digestion of fats in the intestine. In liver, the synthesis of bile acids from cholesterol is regulated by multiple signaling cascades that repress transcription of the gene encoding cholesterol $7 \alpha$-hydroxylase (CYP7A1), the rate-limiting enzyme in the classic bile acid synthesis pathway. In this issue of the JCI, Ito and coworkers demonstrate that mice lacking $\beta$ Klotho, a membrane protein with 2 putative glycosidase domains, have increased Cyp7a1 mRNA levels and bile acid concentrations (see the related article beginning on page 2202). $\beta \mathrm{Klotho}-\mathrm{KO}$ mice also have small gallbladders and are resistant to cholesterol gallstone formation. These findings highlight the central role of $\beta$ Klotho in bile acid homeostasis and raise the possibility that this protein could be a pharmacologic target for the treatment of gallstones.

Nonstandard abbreviations used: CYP7A1, cholesterol $7 \alpha$-hydroxylase; FGFR4, fibroblast growth factor receptor 4; FXR, farnesoid X receptor; SHP, small heterodimer partner.

Conflict of interest: The authors have declared that no conflict of interest exists.

Citation for this article: J. Clin. Invest. 115:2075-2077 (2005). doi:10.1172/JCI26046.
Bile acids are cholesterol metabolites that are synthesized in the liver, stored in the gallbladder, and released during a meal into the small intestine, where they are crucial for the absorption of lipophilic nutrients and vitamins (1). Despite their importance in normal physiology, bile acids are strong detergents whose con- centrations must be tightly controlled. Dysregulation of bile acid homeostasis is associated with a range of pathophysiological disorders including cholestatic liver disease and cholesterol gallstone formation. In this issue of the JCI, Ito et al. make an interesting and unexpected link between the protein $\beta$ Klotho and the regulation of bile acid synthesis (2).

\section{Feedback repression of bile acid synthesis}

Cholesterol $7 \alpha$-hydroxylase (CYP7A1), which is encoded by the CYP7A1 gene, catalyzes the rate-limiting step in the conversion of cholesterol to bile acids (Figure 1). Bile acids act via a feedback mechanism to repress CYP7A1 transcription. Work from a number of laboratories has revealed the complexity of this regulation $(1,3)$. One pathway through which CYP7A1 is 


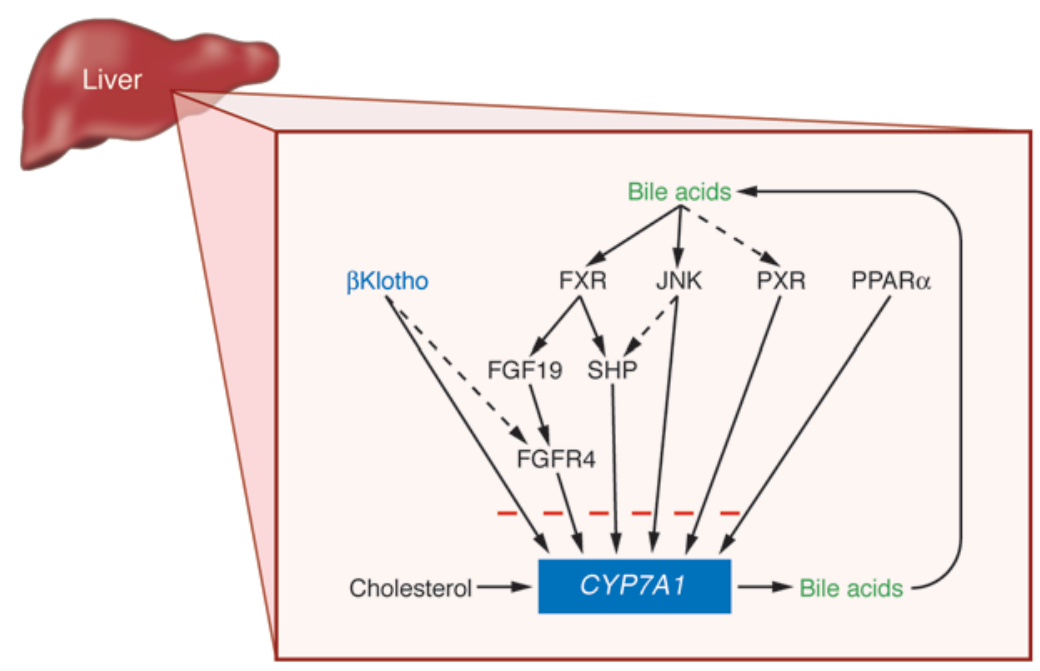

\section{Figure 1}

Shown are signaling pathways that repress transcription of the gene encoding CYP7A1, the rate-limiting enzyme in bile acid synthesis. Established pathways for the repression of Cyp7a1 in mice are indicated by solid lines. These include JNK and the nuclear bile acid receptor FXR. Activation of FXR represses CYP7A1 by induction of the orphan nuclear receptor SHP and FGF19, which acts through FGFR4. Activation of the nuclear receptors pregnane $X$ receptor (PXR) and PPAR $\alpha$ by xenobiotics and fatty acids, respectively, also represses CYP7A1 through unknown mechanisms. Speculative pathways are shown as dotted lines. In this issue, Ito and colleagues show that mice lacking $\beta$ Klotho have increased Cyp7a1 expression and bile acid synthesis and are resistant to the formation of gallstones (2). The similar phenotypes of $\beta$ Klotho-KO and FGFR4-KO mice (8), including increased bile acid synthesis and small gallbladders, suggest that these proteins may act through a common pathway. repressed involves the nuclear bile acid receptor farnesoid $X$ receptor (FXR). Activation of FXR represses CYP7A1 indirectly through induction of the orphan nuclear receptor small heterodimer partner (SHP), which then binds to another orphan nuclear receptor, liver receptor homolog-1, in the CYP7A1 gene promoter to repress gene transcription. Mice lacking FXR or SHP have increased Cyp7a1 expression and a corresponding increase in bile acid synthesis (4-7). FXR also induces the expression of FGF19 in human hepatocytes, which represses CYP7A1, presumably in an autocrine or paracrine fashion (8). Mice lacking fibroblast growth factor receptor 4 (FGFR4), a receptor for FGF19, have increased Cyp7a1 mRNA levels and a corresponding increase in bile acid synthesis (8). Thus, FXR contributes to the repression of Cyp7a1 through multiple mechanisms.

However, FXR-mediated suppression does not constitute the whole story when it comes to feedback regulation of CYP7A1. Treatment of SHP-KO and FGFR4-KO mice with pharmacologic doses of bile acids still results in repression of Cyp7a1, which reveals the presence of additional regulatory mechanisms $(5,6,9)$. One of the FXR/SHPindependent pathways involves activation of liver Kupffer cells, which secrete inflammatory cytokines such as TNF- $\alpha$ (10). These cytokines repress Cyp7a1 in hepatocytes through a signaling pathway that involves JNK (11). The nuclear receptors pregnane $X$ receptor and PPAR $\alpha$, which are activated by xenobiotics and fatty acids, respectively, also repress Cyp7a1 through mechanisms that do not appear to involve FXR or SHP (3). Thus, multiple regulatory pathways converge to repress Cyp7a1 (Figure 1).

\section{$\beta$ Klotho regulates bile acid homeostasis}

Klotho made a dramatic entrance several years ago when it was shown that mice with reduced levels of this protein have a premature aging phenotype that includes dysregulation of calcium and phosphorus homeostasis (12). Recently, a close relative, termed $\beta$ Klotho, was shown to be expressed in liver, pancreas, and fat (13). Both Klotho and $\beta$ Klotho are membrane proteins containing 2 regions in the extracellular domain with homology to those in family 1 glycosidases, which hydrolyze glycosidic bonds. To gain insight into $\beta$ Klotho's function, Ito and colleagues disrupted the gene encoding $\beta$ Klotho in mice (2). Notably, $\beta$ Klotho$\mathrm{KO}$ mice have pronounced alterations in bile acid metabolism, including marked increases in Cyp7a1 mRNA levels and a corresponding increase in bile acid excretion in the feces. These changes are consistent with disruption of the normal bile acid feedback regulatory loop (Figure 1).

It is not clear how $\beta$ Klotho contributes to the repression of Cyp7a1. Although bile acidmediated induction of hepatic Shp mRNA was reduced in the $\beta$ Klotho-KO mice, there was no significant difference in basal Shp mRNA levels in $\beta$ Klotho $\mathrm{KO}$ and wild-type mice (2). These data suggest that $\beta$ Klotho-mediated repression of Cyp7a1 does not involve an increase in SHP concentrations. Studies with selective FXR agonists in the $\beta$ Klotho-KO mice are needed to clarify whether $\beta$ Klotho is required for FXR to repress Cyp7a1.

One intriguing possibility is that $\beta$ Klotho works in concert with FGFR4. Both are highly expressed in liver, and the $\beta$ Klotho-KO and FGFR4-KO mice have remarkably similar phenotypes, including elevated Cyp7a1 mRNA levels and bile acid synthesis as well as a small gallbladder $(2,8)$. Interestingly, heparin/heparan sulphate-like glycosaminoglycans serve as cofactors for FGF signaling through FGFRs, which raises the possibility that $\beta$ Klotho, with its 2 putative glycosidase domains, might regulate the concentrations or activity of a cofactor required for FGFR4 signaling.

\section{Absence of $\beta$ Klotho protects against gallstones}

What are the potential clinical implications of this work? Ito et al. (2) show that $\beta$ Klotho$\mathrm{KO}$ mice are resistant to the formation of gallstones, which develop when the ratio of cholesterol to bile acids and phospholipids in the bile increases to the point that cholesterol precipitates (14). In mice, cholesterol gallstone formation can be induced by a lithogenic diet that includes high cholesterol and cholic acid concentrations (15). The authors suggest that the lack of gallstone formation in $\beta$ Klotho-KO mice could be due to the increase in hepatic bile acid synthesis, which may alter the cholesterol/bile acid ratio in bile to reduce gallstone formation. However, the lipid composition of the bile in the $\beta$ Klotho-KO mice was not tested directly nor were the hepatic protein and mRNA levels determined for bile salt export pump (BSEP; also known as ABCB11) and multidrug-resistance protein 2 (also known as ABCB4), which transport bile acids and phosphatidylcholine into the bile $(16,17)$. It is noteworthy that an increase in biliary bile acid secretion was not sufficient to prevent cholesterol gallstone formation in transgenic mice overexpressing the bile acid trans- 
porter BSEP (18). Thus, the resistance of $\beta$ Klotho-KO mice to gallstones is likely to be a consequence of more than just increased bile acid concentrations in the bile.

One possibility is that the small size of the gallbladder in the $\beta$ Klotho-KO mice reflects changes that contribute to the prevention of gallstone formation. An increase in gallbladder contraction and emptying would be expected to reduce the time that cholesterol has to precipitate and form stones in the bile (19). Indeed, gallbladder stasis is associated with gallstone formation in both humans and rodents $(20$, 21). It will be interesting to learn whether $\beta \mathrm{Klotho}$ is present in the gallbladder and how it affects gallbladder function. Moreover, it will be interesting to see whether FGFR4-KO mice, which also have small gallbladders, are resistant to gallstones.

In summary, the work of Ito and colleagues (2) adds $\beta$ Klotho to the complex tapestry of signaling networks that regulate bile acid homeostasis. Precisely how $\beta$ Klotho integrates with these other regulatory pathways remains to be determined.

Address correspondence to: Steven A. Kliewer, University of Texas Southwestern Medical Center, 5323 Harry Hines Boulevard, Room ND9.502, Dallas, Texas 753909041, USA. Phone: (214) 645-6304; Fax:
(214)645-6305; E-mail: steven.kliewer@ utsouthwestern.edu.

1. Russell, D.W. 2003. The enzymes, regulation, and genetics of bile acid synthesis. Annu. Rev. Biochem. 72:137-174.

2. Ito, S., et al. 2005. Impaired negative feedback suppression of bile acid synthesis in mice lacking BKlotho. J. Clin. Invest. 115:2202-2208. doi:10.1172/ JCI23076.

3. Chiang, J.Y. 2004. Regulation of bile acid synthesis: pathways, nuclear receptors, and mechanisms [review]. J. Hepatol. 40:539-551.

4. Sinal, C.J., et al. 2000. Targeted disruption of the nuclear receptor FXR/BAR impairs bile acid and lipid homeostasis. Cell. 102:731-744.

5. Kerr, T.A., et al. 2002. Loss of nuclear receptor SHP impairs but does not eliminate negative feedback regulation of bile acid synthesis. Dev. Cell. 2:713-720.

6. Wang, L., et al. 2002. Redundant pathways for negative feedback regulation of bile acid production. Dev. Cell. 2:721-731.

7. Kok, T., et al. 2003. Enterohepatic circulation of bile salts in farnesoid $X$ receptor-deficient mice: efficient intestinal bile salt absorption in the absence of ileal bile acid-binding protein. J. Biol. Chem. 278:41930-41937.

8. Holt, J.A., et al. 2003. Definition of a novel growth factor-dependent signal cascade for the suppression of bile acid biosynthesis. Genes Dev. 17:1581-1591.

9. Yu, C., et al. 2000. Elevated cholesterol metabolism and bile acid synthesis in mice lacking membrane tyrosine kinase receptor FGFR4. J. Biol. Chem. 275:15482-15489.

10. Davis, R.A., Miyake, J.H., Hui, T.Y., and Spann, N.J. 2002. Regulation of cholesterol-7alpha-hydroxylase: BAREly missing a SHP [review]. J. Lipid Res. 43:533-543.

11. Gupta, S., Stravitz, R.T., Dent, P., and Hylemon, P.B. 2001. Down-regulation of cholesterol 7alphahydroxylase (CYP7A1) gene expression by bile acids in primary rat hepatocytes is mediated by the c-Jun $\mathrm{N}$-terminal kinase pathway. J. Biol. Chem. 276:15816-15822.

12. Kuro-o, M., et al. 1997. Mutation of the mouse klotho gene leads to a syndrome resembling ageing. Nature. 390:45-51.

13. Ito, S., et al. 2000. Molecular cloning and expression analyses of mouse betaklotho, which encodes a novel Klotho family protein. Mech. Dev. 98:115-119.

14. Admirand, W.H., and Small, D.M. 1968. The physicochemical basis of cholesterol gallstone formation in man. J. Clin. Invest. 47:1043-1052.

15. Tepperman, J., Caldwell, F.T., and Tepperman, H.M. 1964. Induction of gallstones in mice by feeding a cholesterol-cholic acid containing diet. Am.J. Physiol. 206:628-634.

16. Gerloff, T., et al. 1998. The sister of P-glycoprotein represents the canalicular bile salt export pump of mammalian liver. J. Biol. Chem. 273:10046-10050.

17. Smit, J.J., et al. 1993. Homozygous disruption of the murine mdr2 P-glycoprotein gene leads to a complete absence of phospholipid from bile and to liver disease. Cell. 75:451-462.

18. Figge, A., et al. 2004. Hepatic overexpression of murine Abcb11 increases hepatobiliary lipid secretion and reduces hepatic steatosis. J. Biol. Chem. 279:2790-2799.

19. Shaffer, E.A. 1992. Abnormalities in gallbladder function in cholesterol gallstone disease: bile and blood, mucosa and muscle - the list lengthens. Gastroenterology. 102:1808-1812.

20. van Erpecum, K.J., et al. 1992. Fasting gallbladder volume, postprandial emptying and cholecystokinin release in gallstone patients and normal subjects. J. Hepatol. 14:194-202.

21. Wang, D.Q., Schmitz, F., Kopin, A.S., and Carey, M.C. 2004. Targeted disruption of the murine cholecystokinin-1 receptor promotes intestinal cholesterol absorption and susceptibility to cholesterol cholelithiasis. J. Clin. Invest. 114:521-528. doi:10.1172/JCI200416801.

\title{
A molecule's right to choose: how diabetogenic class II MHC products bind peptides
}

\author{
Hidde L. Ploegh \\ Department of Pathology, Harvard Medical School, Boston, Massachusetts, USA.
}

\begin{abstract}
The distinction between peptides that bind to class II MHC products under laboratory conditions and those that do so physiologically is important for the prediction of antigens recognized by autoreactive $T$ cells. In this issue of the JCI, Suri et al., using antigen-presenting cells, compared the peptides that bound to human HLA-DQ8 and those that bound to mouse I-A $\mathrm{A}^{\mathrm{7}}$, both class II MHC products that predispose their carriers to type 1 diabetes (see the related article beginning on page 2268). The rules of engagement for the peptide ligands of the DQ8 and $\mathrm{I}-\mathrm{A}^{\mathrm{g} 7}$ molecules involve similarities in their anchor residues, which mediate stable interaction with class II MHC products. The peptides identified derive from overlapping sets of self proteins.
\end{abstract}

Nonstandard abbreviations used: P9, position 9.

Conflict of interest: The author has declared that no conflict of interest exists.

Citation for this article: J. Clin. Invest. 115:2077-2079 (2005). doi:10.1172/JCI26018.
The single most important genetic factor that predisposes to autoimmune disease is the MHC. In the case of type 1 diabetes, individuals who carry the MHC class II HLA-DQ8 allele are more likely to contract the disease than those who do not, and other alleles at the class II loci (HLA-DR3, HLA-DR4) can predispose to this disease as well. The predominant contribution of the MHC to disease susceptibility is mirrored in the available animal models of autoimmune disease, including diabetes.

In the absence of experiments conducted on humans, the appropriateness of various animal models as stand-ins for human disease continues to be called into question. In mice and humans, the MHC products are very similar in their 3-dimensional structure, although they are no more than $70 \%$ identical in amino acid sequence. Different residues constitute the peptide-bind- 